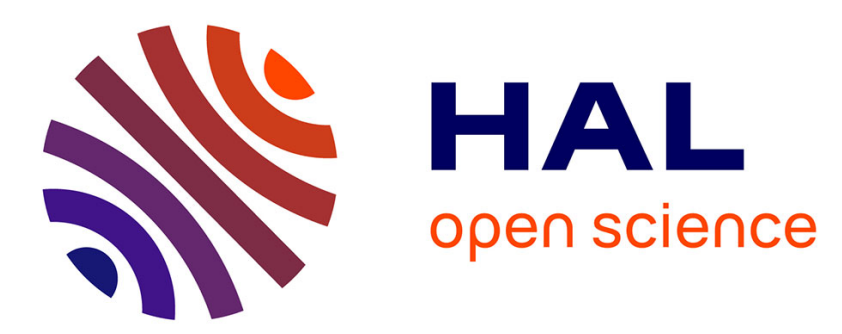

\title{
Numerically exact quantum dynamics of bosons with time-dependent interactions of harmonic type
}

Axel Lode, Kaspar Sakmann, Ofir Alon, Lorenz Cederbaum, Alexej Streltsov

\section{To cite this version:}

Axel Lode, Kaspar Sakmann, Ofir Alon, Lorenz Cederbaum, Alexej Streltsov. Numerically exact quantum dynamics of bosons with time-dependent interactions of harmonic type. Physical Review A : Atomic, molecular, and optical physics [1990-2015], 2012, 86 (6), 10.1103/PhysRevA.86.063606 . hal-02369797

\section{HAL Id: hal-02369797 https://hal.science/hal-02369797}

Submitted on 19 Nov 2019

HAL is a multi-disciplinary open access archive for the deposit and dissemination of scientific research documents, whether they are published or not. The documents may come from teaching and research institutions in France or abroad, or from public or private research centers.
L'archive ouverte pluridisciplinaire HAL, est destinée au dépôt et à la diffusion de documents scientifiques de niveau recherche, publiés ou non, émanant des établissements d'enseignement et de recherche français ou étrangers, des laboratoires publics ou privés. 


\title{
Numerically exact quantum dynamics of bosons with time-dependent interactions of harmonic type
}

\author{
Axel U. J. Lode, ${ }^{1}$ Kaspar Sakmann, ${ }^{1}$ Ofir E. Alon, ${ }^{2}$ Lorenz S. Cederbaum, ${ }^{1}$ and Alexej I. Streltsov ${ }^{1, *}$ \\ ${ }^{1}$ Theoretische Chemie, Physikalisch-Chemisches Institut, Universität Heidelberg, Im Neuenheimer Feld 229, D-69120 Heidelberg, Germany \\ ${ }^{2}$ Department of Physics, University of Haifa at Oranim, Tivon 36006, Israel
}

(Received 20 July 2012; published 6 December 2012)

\begin{abstract}
The exactly solvable quantum many-particle model with harmonic one- and two-particle interaction terms is extended to include time dependency. We show that when the external trap potential and interparticle interaction have a time dependency, the numerically exact solutions of the corresponding time-dependent many-boson Schrödinger equation are still available. We use these exact solutions to benchmark the recently developed multiconfigurational time-dependent Hartree method for bosons (MCTDHB) [Phys. Rev. Lett. 99, 030402 (2007); Phys. Rev. A 77, 033613 (2008)]. In particular, we benchmark the MCTDHB method for (i) the ground state; (ii) the breathing many-body dynamics activated by a quench scenario where the interparticle interaction strength is suddenly turned on to a finite value; (iii) the nonequilibrium dynamic for driven scenarios where both the trap- and interparticle-interaction potentials are time-dependent. Excellent convergence of the ground state and dynamics is demonstrated. The great relevance of the self-consistency and time adaptivity, which are the intrinsic features of the MCTDHB method, is demonstrated by contrasting the MCTDHB predictions and those obtained within the standard full configuration interaction method spanning a Fock space of the same size, but utilizing as one-particle basis set the fixed-shape eigenstates of the one-particle potential. Connections of the model's results to ultracold Bose-Einstein condensed systems are addressed.
\end{abstract}

DOI: 10.1103/PhysRevA.86.063606

PACS number(s): 03.75.Kk, 05.30.Jp, 03.65.-w

\section{INTRODUCTION}

Since the first realizations of Bose-Einstein condensates (BECs) [1-3] the experiments on this unique state of quantum systems have become more and more complex. Nowadays, the strength of the interparticle interactions, the trapping potential, and the dimensionality of BECs are under experimental control [4-8]. This makes BECs a vivid and rich testing ground for a wide range of physical theories. Recent realizations of dipolar BECs [9-11] open a new perspective in the research of the physics of ultracold atoms and molecules. A control on a new degree of freedom is achieved-the dipolar long-range part of the interparticle interaction can now be customized. This achievement can be considered as a first successful step towards a control on the overall shape of the interparticle interaction. It also stimulates the development of theoretical methods capable of solving the time-(in)dependent many-particle Schrödinger equation (TDSE) which governs the physics of the trapped ultracold systems with general interparticle interactions. The class of many-body Hamiltonians which permits analytical solutions is quite small, so, generally, one has to rely on numerical many-body methods to solve the TDSE. The many-body methods in use have to be qualified to describe quantum many-body statics and dynamics. Benchmarking of these methods against exactly solvable Hamiltonians is a necessary step for such a qualification.

In this work we consider an exactly solvable many-body Hamiltonian, where both the one-body (trap) and two-body (interparticle interaction) potentials are of harmonic type, also known as the harmonic interaction model (HIM) (see Refs. [12,13]). The exact solutions of the HIM problem

\footnotetext{
*Alexej.Streltsov@pci.uni-heidelberg.de
}

are obtained by transformation of the Hamiltonian from the laboratory to the center-of-mass frame, where the Hamiltonian becomes separable. The price of the transformation is that an intuitive physical picture of real particles is lost and, instead, one operates with effective "particles" representing the transformed coordinates. One wants to have, first, a general many-body method for identical physical particles where each particle has its own real coordinate. Second, the method must be powerful enough to solve problems where such real coordinates are not favorable (suitable). Third, it should be capable of solving general problems where separations (transformations) of the variables are impossible, as is the case in unharmonic and multiwell traps. In this work we want to test such a method-the recently developed multiconfigurational time-dependent Hartree for bosons (MCTDHB) method [14,15], which can treat the dynamics, i.e., the TDSE for trapped bosons, with general interparticle interactions. We would like to carry the examples to an extreme case separable in suitable coordinates; in this way we can unambiguously test the performance of the MCTDHB against analytically and/or numerically exact solutions.

While there are several known many-body models with time-independent Hamiltonians which have analytical solutions, exactly solvable time-dependent many-body Hamiltonians are even less abundant. Why does one need to study them? Apart from an exploration of novel dynamical physical phenomena, there is a practical reason for it. In typical experiments with ultracold systems the manipulations of the trapping potentials, as well as altering of the magnetic field used in the Feshbach resonance technique(s) [7] to manipulate the interparticle interaction, are time-dependent procedures. Hence, there is a need for a proven theoretical method capable of solving time-dependent Hamiltonians where both the trap and interparticle interaction potentials are time dependent. However, the TDSE with 
general time-dependent Hamiltonians can be solved only numerically. Hence it is very difficult to verify and quantify the region of applicability and quality of the numerical solutions obtained. Convincing benchmarks against exact results are of great relevance. In this work we show how to extend the exactly solvable quantum many-particle HIM problem to include time dependency, and use it to benchmark the MCTDHB method.

It is worthwhile to mention that some physical phenomena and properties of the many-body solutions of the HIM problem are "universal," i.e., transferable to systems with other interparticle interactions, e.g., contact interaction. In this respect it is timely to stress the relevance of the physics of the HIM for the field of ultracold atoms. As an analytically solvable many-body model with two-body interaction for the case of attractive bosons, Wilkin et al. studied the question whether they condense in Ref. [16]. In Refs. [17,18] the criterion for Bose-Einstein condensation and the coherence properties of attractive ultracold bosons was studied using the static HIM model. Reference [13] deals with the physics of the ground and excited states of the HIM and their relation to the physics of ultracold atoms. All the mentioned works consider statics. In the present study we consider the interesting dynamics occurring in the HIM model with time-independent and even time-dependent Hamiltonians. For example, small displacements of the density out of the center of a harmonic trap result in so-called dipole oscillations with the trap frequency which are independent of the interparticle interaction. Another example is a quench of the interparticle interaction in a harmonically trapped system - it activates only breathing excitations which preserve the symmetry of the trap. In the present work we discover a time-dependent phenomenon in the extended HIM, and discuss its "universality" for the harmonically trapped systems with general interparticle interactions.

The structure of the paper is as follows. In Sec. II we introduce the harmonic interaction model and discuss the aftermath and implications appearing due to the transformation of the coordinates from the center-of-mass frame, where the exact solutions are analytically known, to the laboratory frame where we want to solve the problem numerically. The MCTDHB method is briefly reviewed in Sec. III. Section IV provides detailed benchmarks and comparisons of the exact and numerical results for the ground state of the HIM obtained within the framework of the MCTDHB and the standard full configuration interaction (FCI) methods; the latter is also known as the exact diagonalization (ED) technique. In Sec. V we benchmark our numerical tools to describe the breathing many-body dynamics activated by a quench scenario where the interparticle interaction strength is suddenly turned on from zero to a finite value. Section VI shows how to extend the exactly solvable quantum many-particle model with harmonic one- and two-particle interaction terms to include time dependency. Here we also demonstrate the applicability of the MCTDHB method to describe numerically exact many-boson dynamics for complicated scenarios where both the external trap and interparticle interaction potentials are time dependent. Section VII summarizes our results and provides an outlook for the predictions obtained for the HIM problem to ultracold atomic systems with contact interactions.

\section{THE HARMONIC INTERACTION MODEL}

\section{A. Basic definitions}

Our starting point is the HIM (see, e.g., Refs. [12,13]). The Hamiltonian of the HIM is readily obtained in the laboratory frame of reference by setting the interparticle interaction potential $\hat{W}$ and the one-body potential $\hat{V}$ in the many-body Hamiltonian in dimensionless units,

$$
\hat{H}=\sum_{i=1}^{N}\left[\hat{T}\left(\vec{r}_{i}\right)+\hat{V}\left(\vec{r}_{i}\right)\right]+\sum_{i<j}^{N} \hat{W}\left(\vec{r}_{i}, \vec{r}_{j}\right),
$$

to be harmonic:

$$
\hat{W}\left(\vec{r}_{i}, \vec{r}_{j}\right)=K_{0}\left(\vec{r}_{i}-\vec{r}_{j}\right)^{2}, \quad \hat{V}(\vec{r})=\frac{1}{2} \omega^{2} \vec{r}^{2} .
$$

Here, $K_{0}$ accounts for the strength of the two-body interaction and $\hat{T}(\vec{r})=-\frac{1}{2} \partial_{\vec{r}}^{2}$ is the kinetic energy operator. A positive value of $K_{0}$ corresponds to an attraction, while a negative value means repulsion. In the case of a parabolic trapping potential, it is easy to see that the system becomes unbound when the value of $K_{0}$ is negative and big enough for the two-body repulsion to overcome the one-body harmonic trapping, i.e., $K_{0}<-\frac{\omega^{2}}{2 N}$.

Following Cohen and Lee in Ref. [12], the Hamiltonian, Eqs. (1) and (2), can be separated into $N$ independent harmonic oscillators by the following coordinate transformations:

$$
\begin{aligned}
\vec{q}_{j} & =\frac{1}{\sqrt{j(j+1)}} \sum_{i=1}^{j}\left(\vec{r}_{j+1}-\vec{r}_{i}\right), \quad j=1, \ldots, N-1, \\
\vec{q}_{N} & =\frac{1}{\sqrt{N}} \sum_{i=1}^{N} \vec{r}_{i} .
\end{aligned}
$$

The transformed Hamiltonian in the center-of-mass frame reads:

$$
\begin{aligned}
\hat{H} & =\hat{H}_{\text {rel }}+\hat{H}_{\text {c.m. }}, \quad \hat{H}_{\text {rel }}=\sum_{i=1}^{N-1}\left(-\frac{1}{2} \partial_{\vec{q}_{i}}^{2}+\frac{1}{2} \delta_{N}^{2} \vec{q}_{i}^{2}\right), \\
\hat{H}_{\text {c.m. }} & =-\frac{1}{2} \partial_{\vec{q}_{N}}^{2}+\frac{1}{2} \omega^{2} \vec{q}_{N}^{2} .
\end{aligned}
$$

Here, $\delta_{N}=\sqrt{\omega^{2}+2 N K_{0}}$ is the trapping frequency of the $N-1$ harmonic oscillators originating from the set of relative coordinates, and one harmonic oscillator with the frequency $\omega$ representing the center-of-mass coordinate. This separability of the HIM Hamiltonian into the center-of-mass and relative coordinates allows the following visualization: The overall HIM system can be pictured as a medium formed by $N-1$ identical, noninteracting particles associated with relative coordinates $q_{k}, k=1, \ldots, N-1$, moving in an effective harmonic trap with a time-independent frequency $\delta_{N}$, and an independent effective particle with coordinate $q_{N}$, representing the system's center of mass, trapped in the original timeindependent harmonic potential with frequency $\omega$.

The general solution of the HIM problem in its separable form, Eq. (4), is a product of $N$ generally different harmonic oscillator wave functions, and the total energy is the sum of the corresponding oscillator's energies. The exact energy $E_{\text {exact }}$ of the ground state takes on a very simple form (see, e.g., 
Refs. [12,13]):

$$
E_{\text {exact }}=\frac{D}{2}(N-1) \delta_{N}+\frac{D}{2} \omega .
$$

Here, $D$ is the dimensionality of the HIM system. We note that the HIM problem is an example of a many-body system with infinite-range interparticle interactions which permits analytical solution in any dimension, i.e., in one dimension and in higher dimensions. This is another attractive feature of the HIM model relevant for benchmarking numerical methods for the many-particle TDSE.

\section{B. Representing the HIM with basis functions}

Let us consider the ground state of the (one-dimensional) HIM problem for $N=2$ bosons. We contrast the solution written in the center-of-mass frame, i.e., in the $q_{1}, q_{2}$ coordinates with that in the laboratory frame, i.e., in $x_{1}, x_{2}$ coordinates. The corresponding transformations of the coordinates are given in Eq. (4) with $\vec{q}_{j}=q_{j}$ and $\vec{r}_{j}=x_{j}$. It is convenient to denote the $n$th harmonic oscillator $(\mathrm{HO})$ function as $\psi_{n}^{\mathrm{HO}}(X, \Omega)$, where $\Omega$ is the harmonic oscillator frequency and $X$ a general variable. The ground-state solution of the two-particle HIM problem reads:

$$
\begin{aligned}
\Psi\left(q_{1}, q_{2}\right) & =\psi_{0}^{\mathrm{HO}}\left(q_{1}, \delta_{2}\right) \psi_{0}^{\mathrm{HO}}\left(q_{2}, \omega\right)=\mathcal{N} e^{-(1 / 2) \delta_{2} q_{1}^{2}} e^{-(1 / 2) \omega q_{2}^{2}} \\
\Psi\left(x_{1}, x_{2}\right) & =\mathcal{N} e^{-(1 / 2) \delta_{2}\left\{\left[(1 / \sqrt{2})\left(x_{2}-x_{1}\right)\right]\right\}^{2}} e^{-(1 / 2) \omega\left[(1 / \sqrt{2})\left(x_{2}+x_{1}\right)\right]^{2}} \\
& =\mathcal{N} e^{-\left[\left(\delta_{2}+\omega\right) / 4\right] x_{2}^{2}} e^{-\left[\left(\delta_{2}+\omega\right) / 4\right] x_{1}^{2}} e^{-(1 / 2)\left(\omega-\delta_{2}\right) x_{2} x_{1}}=\mathcal{N} \psi_{0}^{\mathrm{HO}}\left(x_{1}, \frac{\omega+\delta_{2}}{2}\right) \psi_{0}^{\mathrm{HO}}\left(x_{2}, \frac{\omega+\delta_{2}}{2}\right) e^{-(1 / 2)\left(\omega-\delta_{2}\right) x_{2} x_{1}} \\
& =\mathcal{N} \psi_{0}^{\mathrm{HO}}\left(x_{1}, \frac{\omega+\delta_{2}}{2}\right) \psi_{0}^{\mathrm{HO}}\left(x_{2}, \frac{\omega+\delta_{2}}{2}\right)\left(1-\frac{1}{2}\left(\omega-\delta_{2}\right) x_{2} x_{1}+\frac{1}{8}\left(\omega-\delta_{2}\right)^{2} x_{2}^{2} x_{1}^{2}-\cdots\right) \\
& =\sum_{i \geqslant j=0}^{\infty} a_{i j} \hat{\mathcal{S}} \psi_{i}^{\mathrm{HO}}\left(x_{1}, \frac{\omega+\delta_{2}}{2}\right) \psi_{j}^{\mathrm{HO}}\left(x_{2}, \frac{\omega+\delta_{2}}{2}\right)
\end{aligned}
$$

Here we use the Taylor expansion for the cross term $e^{-(1 / 2)\left(\omega-\delta_{2}\right) x_{2} x_{1}} \cdot \hat{\mathcal{S}}$ is the symmetrization operator and $a_{i j}$ are the known reexpansion coefficients. A close inspection of the above transformation shows that a single Hartree product of two HO wave functions written in the center-of-mass frame is represented by an infinite sum of different Hartree products in the laboratory frame even if one uses the "dressed" frequency $\frac{\omega+\delta_{2}}{2}$. For bosonic systems these Hartree products have to be properly symmetrized to take into account permutational symmetry of the total wave function. Hence, a numerical solution of the HIM Hamiltonian in the laboratory frame is a very involved problem - the numerical convergence depends on how fast and efficiently this sum is spanned.

For numerical treatments the infinite sums, like in (6), must be truncated. The number of the terms $N_{\text {conf }}$ considered defines the size of the Fock space spanned, i.e., the size of the respective secular matrix to be diagonalized in order to find the respective eigenvalues and eigenstates. For a general $N$-boson system this size is $N_{\text {conf }}=\left(\begin{array}{c}N+M-1 \\ N\end{array}\right)$, where $M$ is the number of one-particle functions (orbitals) used to build the symmetrized Hartree products. The two-boson problem can be diagonalized by taking a lot of basis functions, while already for a ten-boson problem with $M=16, N_{\text {conf }}=3,268,760$. This means that the diagonalization of the respective secular matrix is hence not a simple task. Due to this binomial dependency of the size of the spanned Fock subspace, a bosonic system with large number of particles can be tackled only with quite a few orbitals, for example, for $N=1000$ and $M=3$, the size of the secular problem is $N_{\text {conf }}=501,501$, while already for $M=4$ it is $N_{\text {conf }}=167,668,501$. One of the main goals of the present work is to verify that the choice of the basis functions (orbitals) used to build up the permanents (symmetrized Hartree products) has an enormous impact on the numerical convergence of many-body problems.

The above considered analysis of the interplay between the exact solution written in the center of mass and in the laboratory frames of reference is of course applicable for an arbitrary number of particles. The Hartree products appearing in the solutions of the corresponding HIM problems written in the laboratory frame are built up of $\mathrm{HO}$ basis functions with exponents $-\frac{\omega+\delta_{N}}{4} x_{j}^{2}$, i.e., they depend via $\delta_{N}$ on the number of particles $N$ and on the interparticle interaction strength $K_{0}$. Hence, to solve the HIM problem for different parameters $N$ and $K_{0}$ in the laboratory frame it is advantageous to use one-particle basis functions with different shapes (exponents). It is natural to ask the following questions: What to do in a general case, when the analytic solution of the problem is not available, i.e., which basis set to use? And how to find the optimal self-consistent orbitals? The simplest answer is to use the one-particle functions (bare orbitals) of the studied system when the two-body interparticle interactions are switched off. These fixed-shape basis functions are obtained as solutions of the one-particle problem $\hat{h} \psi_{i}=e_{i} \psi_{i}$ with $\hat{h}=\hat{T}(\vec{r})+\hat{V}(\vec{r})$. In the studied HIM it means to use the HO basis $\psi_{n}^{\mathrm{HO}}(x, \Omega)$ with trapping frequency $\Omega=\omega$. The answer to the second question is also known-to use the recently developed MCTDHB method [14,15], which utilizes the Dirac-Frenkel variational principle to determine the optimal shapes of the orbitals for time-dependent problems. In this work we will examine and contrast the performance of two many-body methods to attack the time-dependent and time-independent HIM problems - the full configuration interaction (exact diagonalization) method which utilizes as a basis set the solutions of the one-particle in the harmonic trap, and the self-consistent MCTDHB method. 


\section{THE MCTDHB METHOD}

Let us briefly describe the MCTDHB theory; for complete derivations and some recent applications see the literature [14,15,19-28]. The MCTDHB method has been developed to solve the time-(in)dependent many-boson Schrödinger equation. It relies on a multiconfigurational ansatz for the wave function, i.e., $|\Psi(t)\rangle=\sum_{\vec{n}} C_{\vec{n}}(t)|\vec{n} ; t\rangle$. The unknown $C_{\vec{n}}(t)$ are called expansion coefficients. The permanents $|\vec{n} ; t\rangle$ are built as symmetrized Hartree products of $N$ unknown orthogonal one-particle functions. For $M$ orbitals the number of these permanents is equal to the number all possible permutations of $N$ particles over $M$ orbitals, namely, $\left({ }_{N}^{N+M-1}\right)$. It is noteworthy that both the coefficients $C_{\vec{n}}(t)$ and the one-particle functions used to build the permanents are time-dependent, variationally optimized quantities, which are determined by solving the corresponding MCTDHB equations of motion $[14,15]$. These equations depend on the parameters of the Hamiltonian, on the number of particles as well as on the number of one-particle basis functions used. For different evolution times the optimal orbitals have different shapesthis feature is called time adaptivity.

Within the MCTDHB method the time-independent variational solutions are obtained by propagating the MCTDHB equations in imaginary time, which is equivalent to solving the stationary problem variationally, as developed in the multiconfigurational Hartree method (MCHB) for bosons (see Ref. [29]). Hence, the static solutions we give here qualify as test suits for how the standard (time-independent) variational principle is handled numerically by MCTDHB. From now on we call the time-dependent variational MCTDHB solution "time adaptive" to distinguish it from the "selfconsistent" static, i.e., time-independent MCTDHB solution. If the one-particle functions used are not allowed to be optimized, the MCTDHB method boils down to the standard full configuration interaction (exact diagonalization) method. Thus, one can consider the MCTDHB method as an exact diagonalization method with time-adaptive (self-consistent) orbitals. For a given number of orbitals the dimension of the secular problem involved for the FCI(ED) and MCTDHB computations is the same, $N_{\text {conf }}=\left(\begin{array}{c}N+M-1 \\ N\end{array}\right)$. If only one self-consistent orbital is considered, the MCTDHB theory boils down to the famous Gross-Pitaevskii (GP) mean-field theory widely and often successfully used to describe statics and dynamics of condensed bosonic systems [30-32].

At this point it is very important to stress that the MCTDHB and standard FCI methods used in this work operate with general Hamiltonians in the laboratory frame of reference. Hence the separability of the HIM is not taken into account. But this formulation of the methods allows one to attack general, i.e., inseparable problems as has been done in, e.g., Refs. [21, 22,24-28].

The MCTDHB equations of motion are solved numerically efficiently with the MCTDHB program package [19]. The current study relies on the propagation of the orbitals' equations of motion with a shared-memory parallelized implementation of the Adams-Bashforth Moulton predictor corrector integrator [33] and the coefficients' equations of motion with a hybridly OpenMP-MPI parallelized short iterative Lanczos algorithm [34]. As primitive basis functions representing the self-consistent (time-dependent) orbitals, we use either the HO discrete variable representation [35] or the fast Fourier transform collocation method utilizing hybrid OpenMP-MPI parallelization (see [19]).

\section{GROUND STATE OF THE HIM: MCTDHB AND FCI VS EXACT SOLUTION}

We begin by benchmarking the MCTDHB and FCI methods against the ground state of the one-dimensional HIM. We consider systems of $N=2,10,50,100,1000$ bosons trapped in the parabolic trap potential $V(x)=\frac{1}{2} x^{2}$ with the interboson interaction strengths selected to keep $\Lambda=K_{0}(N-1)=0.5$ constant. Such a choice of the interaction strengths implies that all these systems have the same GP solution, i.e., are equivalent at the mean-field level of description. To find the properties of convergence of the MCTDHB and FCI methods towards the exact solution of the HIM, it is instructive to successively increase the number of orbitals $M$ used in the computation. In Fig. 1 we plot the relative difference between the ground-state eigenenergy and the corresponding exact energy $\left(E_{\mathrm{MB}}-E_{\text {exact }}\right) / E_{\text {exact }}$ as a function of the number of orbitals $M$ used. The self-consistent many-body (MB) $\operatorname{MCTDHB}(M)$ results are plotted by open symbols; the corresponding fixed-orbital $\mathrm{FCI}(M)$ results are depicted by filled symbols.

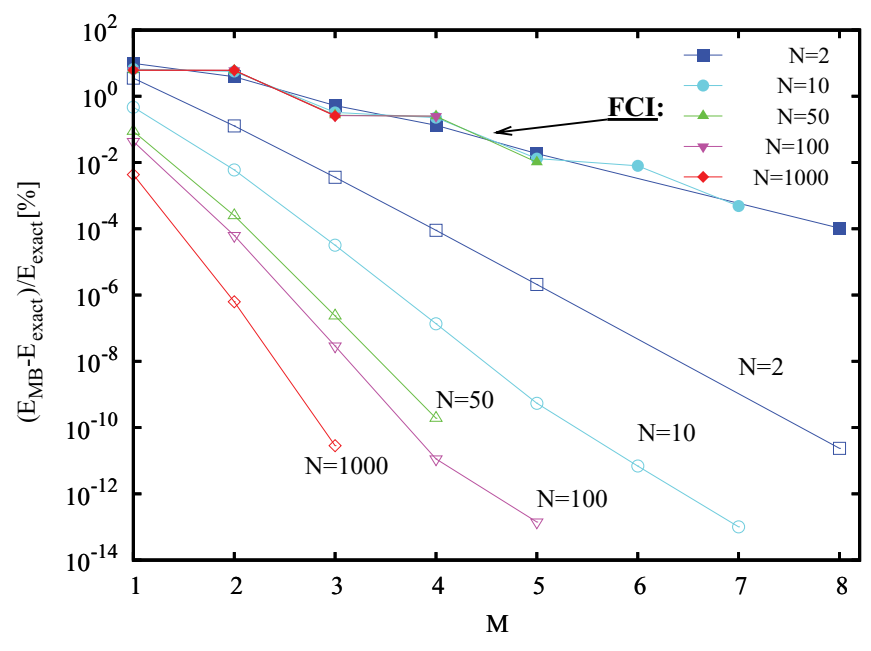

FIG. 1. (Color online) Numerical convergence of the selfconsistent MCTDHB and fixed-orbital FCI (ED) methods for the ground-state energy of the HIM. Systems with $N=2,10,50,100$, and 1000 bosons are considered; the strengths of the interparticle interactions $K_{0}$ have been chosen to keep $\Lambda=K_{0}(N-1)=0.5$ constant. We plot the relative differences between the total energies computed using the MCTDHB (filled symbols) and FCI (ED) (open symbols) many-body methods and respective exact energies in percents, $100\left(E_{\mathrm{MB}}-E_{\text {exact }}\right) / E_{\text {exact }}$, for different orbital number $M$. For a given $M$ both many-body methods span the same Fock space, i.e., the respective secular matrices to be diagonalized are of the same size. The advantage of the appropriate, i.e., self-consistent, choice of the one-particle basis functions is evident-the self-consistent MCTDHB method converges much faster than the fixed-orbital FCI (ED) one. Note the logarithmic scale and number of decades spanned. All quantities shown are dimensionless. 
The key observation seen in Fig. 1 is that the numerical results converge towards the exact ones with increasing the number of the orbitals used. The performance of the selfconsistent MCTDHB method, however, by far exceeds that of the fixed-orbital FCI. Note the logarithmic scale and number of decades spanned. The proper choice of the many-body basis set is very crucial - within the same size of the Fock subspace spanned (dimension of the secular matrix $N_{\text {conf }}$ ) one can get an improvement of about six to eight orders of magnitude. The results prove that the exact solutions of the HIM can be obtained numerically using the MCTDHB method with just a few self-consistent orbitals.

Another striking feature of the MCTDHB method is its performance for different particle numbers. The convergence is faster for larger particle numbers at fixed $\Lambda=K_{0}(N-$ $1)$. This is anticipated, because at the mean-field GP $\equiv$ MCTDHB(1) level the considered systems of bosons are equivalent and the GP solution of the static HIM problem tends to the exact one in the thermodynamic $(N \rightarrow \infty)$ limit. Nevertheless, for a large, but finite number of bosons the MCTDHB significantly improves the GP description. For example, for $N=1000$ the relative differences to the exact energy obtained within the GP and $\operatorname{MCTDHB}(3)$ are $\sim 10^{-3} \%$ and $\sim 10^{-11} \%$ percent, respectively (see Fig. 1). So, the self-consistency becomes more and more relevant for systems made of larger particle numbers. In contrast to the MCTDHB, the fixed-orbital FCI method does not show such a tendency. The poor performance of the FCI (ED) method utilizing the bare HO orbital basis set is evident from the above two-boson analysis in Eq. (6). Instead of the HO eigenfunctions of the trap potential $\psi_{n}^{\mathrm{HO}}(x, \Omega=\omega)$ one has to use the $\mathrm{HO}$ basis functions with "modified" frequency $\Omega=\frac{\omega+\delta_{N}}{2}$. However, in the general case, when an analytical solution is unavailable, the only strict way to find the "proper" basis set is to solve the $\operatorname{MCTDHB}(M)$ equations, which determine variationally the optimal one-particle functions (see Ref. [29]).

To highlight the convergence of the $\operatorname{MCTDHB}(M)$ method with the number of orbitals $M$ used, we present in Table I the total ground-state energies of the above considered systems of $N=10,100,1000$ bosons with $\Lambda=K_{0}(N-1)=0.5$. The exact ground-state energies are from Refs. [12,13] [also see Eq. (5)].

\section{QUENCHING THE INTERPARTICLE INTERACTION: MCTDHB AND FCI VS EXACT RESULTS}

In the previous section we have seen that the numerically exact ground-state solutions of the HIM can be obtained using the MCTDHB method with just a few self-consistent orbitals. The standard FCI (ED) method utilizing the "nonoptimal" fixed-shape orbitals of the noninteracting system has demonstrated a much worse convergence. It makes the usage of the direct diagonalization method with nonoptimal orbitals for large particle numbers impractical. Within the number of orbitals technically allowed to be used (this number defines the size of the respective secular matrix to be diagonalized) the quality of the obtained many-body results is unsatisfactory. They can be worse than the one-orbital self-consistent meanfield (GP) results (see Fig. 1).
Having established the great relevance of self-consistency for statics, in the present section we clarify its impact on the quantum many-boson dynamics. The main difference between statics and dynamics is that quantum dynamics involve a lot of excited states and, therefore, the applied many-body method has to be capable of describing them. Indeed, the evolution of any given initial many-body state is obtained as a solution of the time-dependent Schrödinger equation:

$$
\begin{aligned}
\Psi(t) & =\sum_{j=0}^{\infty} a_{j} e^{-i E_{j} t} \Phi_{j}(\vec{X}) \\
& =\sum_{j=0}^{\infty}\left\langle\Psi_{0}(\vec{X}) \mid \Phi_{j}(\vec{X})\right\rangle e^{-i E_{j} t} \Phi_{j}(\vec{X}) .
\end{aligned}
$$

Here $\Psi_{0}(\vec{X})$ is the initial state and $\Phi_{j}(\vec{X})$ and $E_{j}$ are the eigenstates and respective eigenenergies of the quantum system. $\vec{X}$ are the coordinates of the constituting particles. For the HIM all the eigenstates and respective eigenenergies are known in the center-of-mass frame. Hence, to study the exact evolution of the many-body system one needs to evaluate the overlap integrals $a_{j}=\left\langle\Psi_{0}(\vec{X}) \mid \Phi_{j}(\vec{X})\right\rangle$. When computing with the MCTDHB we, of course, work in the laboratory frame and the time-dependent many-body wave function is a complicated nonterminating expansion in terms of permanents.

Let us study a scenario with the HIM Hamiltonian where the many-body dynamics are activated by a sudden quench of the interparticle interaction strength. It is worthwhile to mention that the MCTDHB method has been successfully used in Ref. [36] to describe such a scenario for ultracold systems with contact interaction. On the experimental side the quench of the interparticle interaction is a routine procedure controlled by the Feshbach resonance technique. We assume that the initial state just before the quench was the ground state of the noninteracting system. What kind of dynamics are anticipated in this case? The initial state, i.e., the ground state of the harmonically trapped system, is symmetric, implying that the one-body density has "gerade" symmetry. The sudden quench of the interparticle interaction cannot break this symmetry. Thus, we expect that a change (quench) of the interparticle interaction leads to breathing dynamics of the system-the many-body wave function changes its shape such that the even symmetry is preserved. This dynamical behavior is general and persists in other many-body systems with symmetric trap potentials as well, e.g., in ultracold systems with contact interactions.

\section{A. Breathing dynamics for $\boldsymbol{N}=\mathbf{2}$ bosons}

We first consider the two-boson HIM system where the interparticle interaction strength $K_{0}$ is suddenly quenched from zero to $K_{0}=0.5$. The exact general eigenstates of the HIM system are products of two $\mathrm{HO}$ wave functions-one describes the motion of the relative $q_{1}=\frac{x_{2}-x_{1}}{\sqrt{2}}$ coordinate, another the motion of the center of mass $q_{2}=\frac{x_{2}+x_{1}}{\sqrt{2}}$. Clearly, the center-of-mass part always has bosonic symmetry-it does not change sign when the coordinates of the particles are permuted. In contrast, the relative part can be either bosonic (symmetric) or fermionic (antisymmetric), depending on the 
TABLE I. Ground-state energies of the harmonic interaction Hamiltonian for the systems of $N=10,100,1000$ bosons. Exact analytical versus numerical $\mathrm{MCTDHB}(M)$ results; $M$ is the number of self-consistent orbitals used. The interparticle interaction strengths have been chosen to keep $\Lambda=K_{0}(N-1)=0.5$ constant. In this case all these systems have the same Gross-Pitaevskii solution, i.e., the same energy per particle. The one orbital $\operatorname{MCTDHB}(M=1)$ theory is equivalent to the Gross-Pitaevskii mean field. It is seen that converged results are obtained with less self-consistent orbitals when increasing the number of particles. The energies shown are in dimensionless units.

\begin{tabular}{|c|c|c|c|}
\hline$M$ & $N=10$ & $N=100$ & $N=1000$ \\
\hline 1 & 7.071067811865483 & 70.71067811865483 & 707.1067811865483 \\
\hline 2 & $7.038 \underline{\underline{769026303168}}$ & $70 . \overline{68016951747168}$ & 707.0764334257315 \\
\hline 3 & $7.0383 \overline{50652406389}$ & $70.68012 \overline{2541218675}$ & $707.0764 \overline{289871865}$ \\
\hline 4 & $7.03834 \overline{48424909910}$ & $70.6801253917 \underline{4549}$ & \\
\hline 5 & $7.0383484153 \underline{49058}$ & $70.680125391737 \underline{62}$ & \\
\hline 6 & $7.03834841531 \overline{1494}$ & & \\
\hline 7 & $7.038348415311 \overline{01 \underline{8}}$ & & \\
\hline$E_{\text {exact }}$ & $7.03834841531101 \overline{1}$ & 70.68012539173752 & 707.0764289869851 \\
\hline
\end{tabular}

parity of the Hermite polynomial involved. For example, the first excited $\mathrm{HO}$ state of the relative part $\psi_{1}^{\mathrm{HO}}\left(q_{1}, \tilde{\omega}\right) \sim$ $H_{1}\left(\sqrt{\tilde{\omega}} q_{1}\right) e^{-\tilde{\omega}\left(q_{1}^{2} / 2\right)} \equiv \mathcal{N} q_{1} e^{-\tilde{\omega}\left(q_{1}^{2} / 2\right)}\left[\tilde{\omega}=\frac{\omega+\delta_{2}}{2}\right]$ is fermionic, because the permutation $x_{1} \leftrightarrow x_{2}$ changes the sign of the $q_{1}=$ $\frac{x_{2}-x_{1}}{\sqrt{2}}$ (first Hermite polynomial) and, therefore, the overall sign of the total wave function $\psi_{1}^{\mathrm{HO}}\left(q_{1}, \tilde{\omega}\right) \psi_{0}^{\mathrm{HO}}\left(q_{2}, \omega\right)$. Using this argumentation one can conclude that all even excited $\mathrm{HO}$ states $\psi_{i}^{\mathrm{HO}}\left(q_{1}, \tilde{\omega}\right)$ with $i=0,2,4, \ldots$ of the relative part are bosonic and all odd ones, i.e., $\psi_{i}^{\mathrm{HO}}\left(q_{1}, \tilde{\omega}\right), i=1,3,5, \ldots$ are fermionic.

Having understood the nature of the bosonic and fermionic solutions of the two-particle HIM problem we are ready to analyze the excitations responsible for the breathing dynamics. The ungerade bosonic excitations of the HIM are activated by odd excitations of the center-of-mass part $\psi_{i}^{\mathrm{HO}}\left(q_{2}, \omega\right)$, which oscillates with the original trap frequency $\omega$. The lowest gerade excitation corresponds to the second excited state of the relative part, the next gerade bosonic excitation to the fourth excited state, and so on. In principle, the next class of the gerade excitations appear as products of the HO solutions corresponding to the second excited state of the center-of-mass motion and every even excitation of the relative motion. In the studied quench dynamics we start from the ground state of the noninteracting system, implying that the center-of-mass motion is in the ground state. Therefore, all excited states for which the center of mass is excited are orthogonal to such an initial state and, hence, do not contribute to the dynamics; this is because the overlap integrals of these states with the initial state are zero. Summarizing, the sudden quench of the interparticle interaction in the HIM leads to breathing dynamics with breathing frequencies $\omega(n)=2 n \sqrt{\omega^{2}+4 K_{0}}$, namely, with the main excitation frequency $\omega(n=1) \equiv \omega_{\text {breath }}$ and all its overtones with $n=2,3,4, \ldots$. These frequencies are obtained as energy differences between the ground and respective excited eigenstates.

We use the MATHEMATICA package [37] to compute the required overlap integrals in Eq. (7) and to get the exact timedependent two-boson wave function. Here we have to mention that instead of the infinite summation, the contributions from the 60 exact lowest-in-energy excited states are taken into account; this is more than sufficient for numerical convergence. Next, the exact one-body density as a function of time is obtained according to its definition: the two-body wave function is multiplied by its complex conjugate and one coordinate is integrated out. In Fig. 2 we plot the exact value of the density at the trap center as a function of time by a bold red line. The first two breathing cycles are depicted in the left panel of this figure; the right panel presents the breathing dynamics at longer propagation times. The numerical $M$-orbital MCTDHB and FCI (ED) results are depicted by bold symbols and dotted lines, respectively.

The first observation from Fig. 2 is that the exact density at the middle of the trap oscillates periodically with the breathing frequency $\omega_{\text {breath }}=2 \sqrt{\omega^{2}+4 K_{0}}$. However, the shape of the oscillation differs from the simple $\sim \cos \left(\omega_{\text {breath }} t\right)$ function plotted to guide the eye by a solid black line. This is the result of the contributions from the overtones originating from the higher excited states. The two-orbital MCTDHB(2) solution provides essentially an exact description of the dynamics till half of the breathing cycle-notice the triangles following the exact results. The three-orbital $\operatorname{MCTDHB}(3)$ results, plotted by filled circles, are on top of the exact curve for the first breathing cycle; small deviations from the exact results become visible at the second breathing cycle. The MCTDHB(4) with four time-adaptive orbitals gives the exact description of the first two breathing cycles. The FCI (ED) dynamics with four fixed-shape orbitals, plotted by a double-dashed line, starts to deviate from the exact results already at very short times. Even the six-orbital FCI (ED) dynamics, depicted by a dashed line, starts to deviate from the exact result after one-third of the first breathing cycle. A quite accurate description of the first two breathing oscillations is only obtained on the eight-orbital FCI (ED) level. Summarizing, to describe the first two breathing oscillations of the two-particle HIM problem one needs either four time-adaptive orbitals [MCTDHB(4)] or eight fixed-shape orbitals $[\mathrm{FCI}(8)]$. The above analysis also shows that with time more time-adaptive orbitals are needed to describe the exact dynamics.

Now we quantify the performance of the MCTDHB and FCI (ED) methods to describe the quantum dynamics at longer times. In the right part of Fig. 2 we plot the oscillations of the density at the middle of the trap at longer times. The exact results are depicted by a bold line, the MCTDHB results are depicted by filled symbols, and the FCI (ED) results are plotted 

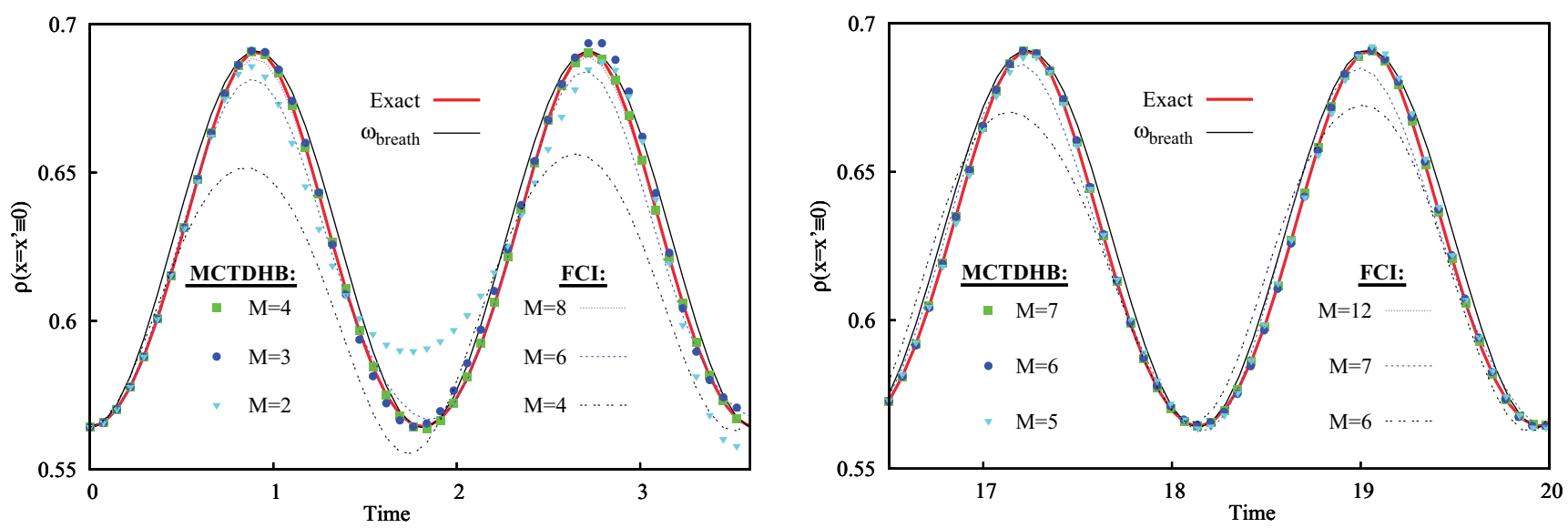

FIG. 2. (Color online) A sudden change (quench) of the interparticle interaction leads to breathing dynamics of the system. We study the HIM system with $N=2$ bosons where the interparticle interaction strength is quenched from zero to $K_{0}=0.5$. The evolution of the one-particle density at the origin $\rho\left(x=x^{\prime} \equiv 0\right)$ is plotted as a function of time. The exact dynamics reveal oscillations namely, with the main breathing frequency $\omega_{\text {breath }}=2 \sqrt{\omega^{2}+4 K_{0}}$ augmented by overtones $2 \omega_{\text {breath }}, 3 \omega_{\text {breath }}, \ldots$ (see text for more details). The solid (black) line depicts the guiding $\sim \cos \left(w_{\text {breath }} t\right)$ function. The numerical MCTDHB and FCI (ED) results are contrasted and compared with the exact ones, plotted by a bold (red) line. The left panel depicts the density oscillations at short times. At the FCI (ED) level an accurate description of the dynamics is achieved by using at least eight fixed-shape orbitals. To gain similar accuracy within the MCTDHB method one needs only three time-adaptive orbitals. Four time-adaptive orbitals $[\operatorname{MCTDHB}(M=4)]$ provide a numerically exact description. The right panel shows the density oscillations at longer times. To describe the dynamics in this case a larger Fock space (more orbitals) is (are) required. A numerically exact description is obtained by using six time-adaptive [MCTDHB(6)] or twelve fixed-shape orbitals [FCI(12)]. See text for further discussion. All quantities shown are dimensionless.

by dashed lines. The exact density continues to oscillate with the main $\omega_{\text {breath }}$ and its overtones. The $\operatorname{MCTDHB}(M>5)$ results are numerically exact. The FCI(12) result, plotted by a dense-dashed line, follows the exact one, while the FCI(6) and FCI(7) are clearly off.

Concluding, for longer propagation time one has to span a larger Fock subspace, i.e., one has to use a larger number of one-particle functions to build the permanents. The difference between the quantum dynamics utilizing fixed-shape and time-adaptive orbital basis sets is clearly seen-to gain a desired accuracy of the propagation one needs to use at least twice as many fixed-shape orbitals than time-adaptive ones. The second important observation is that if a desired convergence of the many-body dynamics is achieved on the $M$-orbital level, further extension of the Fock space is unnecessary; the inclusion of the extra orbitals does not impact the result. This is a general consequence of the variational principle used. It is known for the FCI (ED) method and now proven for the MCTDHB method, which is based on the time-dependent Dirac-Frenkel variational principle. This feature allows us to define a practical strategy for MCTDHB computations. If the dynamics obtained on the $\operatorname{MCTDHB}(M)$ and $\operatorname{MCTDHB}(M+1)$ levels are identical we conclude that numerical convergence to the exact results is reached. In other words, the many-body wave function built from $M$ time-adaptive orbitals is the converged solution of the timedependent many-boson Schrödinger equation.

\section{B. Breathing dynamics for $N=10$ bosons}

Now we examine and compare the performance of the MCTDHB and FCI (ED) methods to treat the time-dependent dynamics of the HIM system with $N=10$ bosons for the same quench scenario as studied before for a system with $N=2$ bosons. By analyzing the structure of the excited states for the $N=10$ system we arrive at the conclusion that a sudden quench leads to the many-body breathing dynamics with frequencies $\omega(n)=2 n \sqrt{\omega^{2}+2 N K_{0}}$, obtained as energy differences between the ground and respective excited eigenstates. Here, the lowest excitation $\omega(n=1) \equiv \omega_{\text {breath }}$ is responsible for the main breathing excitation frequency; higher excited states with $n=2,3,4, \ldots$ result in overtones. The exact results are, in principle, available for systems with any number of particles $[12,13]$. However, the straightforward way of evaluation of the exact time-dependent many-body wave function and the respective density successfully applied for two bosons is much more involved for the ten-particle system. Hence, we employ the numerical MCTDHB method. The first three breathing cycles are depicted in the left part of Fig. 3. The MCTDHB results utilizing $M=8,9,10$ time-adaptive orbitals plotted by dashed lines are indistinguishable from each other. The computational strategy verified and proven above allows us to conclude that the numerically exact description of the TDSE is achieved.

Another important observation seen in Fig. 3 is that the numerically exact MCTDHB results deviate substantially from a simply fitted $\sim \cos \left(\omega_{\text {breath }} t\right)$ curve, plotted by a solid black line to guide the eye. This is a direct evidence that the contribution from higher excited states, responsible for higher overtones, to the breathing dynamics of the $N=10$ boson system is much stronger than it was in the $N=2$ system studied before [compare the exact curve and fit to the $\sim \cos \left(\omega_{\text {breath }} t\right)$ curve in Fig. 2]. The FCI (ED) result with $M=16$ fixed-shape orbitals depicted by filled triangles follows the numerically exact MCTDHB curves only for a very 


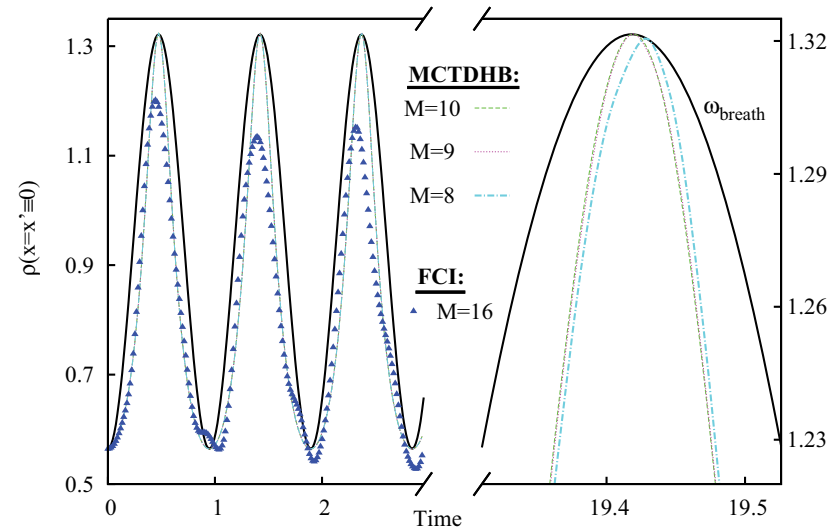

FIG. 3. (Color online) Breathing dynamics of the HIM system with $N=10$ for the same interaction quench scenario as in Fig. 2. The evolution of the one-particle density at the origin $\rho\left(x=x^{\prime} \equiv 0\right)$ is plotted; notice the different scales for the short and long times. The density oscillation is formed by the main breathing frequency $\omega_{\text {breath }}=2 \sqrt{\omega^{2}+2 N K_{0}}$ with strong contributions of the overtones $2 \omega_{\text {breath }}, 3 \omega_{\text {breath }}, \ldots$ (see text for more details). The solid (black) line depicts the guiding $\sim \cos \left(w_{\text {breath }} t\right)$ function. The $\operatorname{MCTDHB}(M=8)$ method with eight time-adaptive orbitals provides a very accurate description of the breathing dynamics for the short and long times. The MCTDHB results for $M=9$ and $M=10$ are identical, indicating that the exact description has been numerically reached. The FCI $(M=16)$ results plotted by triangles start to deviate from the exact solution already for short times. The FCI (ED) method with 16 fixed-shape orbitals provides a reasonable description of the dynamics for a very short time only, i.e., it is incapable of describing more than a half of the first breathing cycle. The exact results could not be obtained in this model from the analytical solution, Eq. (7), because it is more difficult to perform the needed ten-dimensional integrations than to solve the problem numerically exactly by MCTDHB. All quantities shown are dimensionless.

short initial time-for about one-half of the first breathing cycle. For longer propagation times the FCI(16) predictions deviate from the exact results.

The right part of Fig. 3 depicts on an enlarged scale the breathing dynamics at longer times. At all plotted propagation times the eight-orbitals MCTDHB(8) method provides a very accurate description of the many-boson dynamics, while the MCTDHB computations with $M=9,10$ time-adaptive orbitals are numerically exact. We conclude that the usage of time-adaptive orbitals provides an enormous benefit for the accurate description of quantum dynamics of systems with larger particle numbers. In contrast to the MCTDHB method which is capable of providing numerically exact results with a few time-adaptive orbitals, the FCI (ED) treatments even with much larger Fock subspaces spanned cannot provide even a qualitative description of the dynamics.

\section{THE TIME-DEPENDENT HIM: NONEQUILIBRIUM DYNAMICS}

The above discussed visualization of the HIM system as a medium made of $N-1$ noninteracting "relative" particles in which the effective mass particle (representing the center-of-mass coordinate) lives, allows for a simple physical time-dependent generalization. Without loss of separability one can assume that the effective-mass particle moves now not in the stationary but in a time-dependent harmonic potential with driving frequency $\omega(t)$. Moreover, we assume that during this motion the medium representing the relative coordinates remains undisturbed, $\delta_{N}=$ const. Surprisingly, the Hamiltonian corresponding to this problem takes on a simple form in the center-of-mass frame:

$$
\begin{aligned}
\hat{H}(t)= & \hat{H}_{\text {rel }}+\hat{H}_{\text {c.m. }}(t)=\sum_{i=1}^{N-1}\left(-\frac{1}{2} \partial_{\vec{q}_{i}}^{2}+\frac{1}{2} \delta_{N}^{2} \vec{q}_{i}^{2}\right) \\
& -\frac{1}{2} \partial_{\vec{q}_{N}}^{2}+\frac{1}{2} \omega^{2}(t) \vec{q}_{N}^{2} .
\end{aligned}
$$

One can apply reverse engineering and transform this new time-dependent HIM problem back to the laboratory frame:

$$
\hat{H}(t)=\sum_{i=1}^{N}\left[-\frac{1}{2} \partial_{\vec{r}_{i}}^{2}+\frac{1}{2} \omega(t)^{2} \vec{r}_{i}^{2}\right]+K(t) \sum_{i<j}^{N}\left(\vec{r}_{i}-\vec{r}_{j}\right)^{2}
$$

This coupled time-dependent Hamiltonian corresponds to the situation where all real particles are trapped in the time-dependent potential $\hat{V}(\vec{r}, t)=\frac{1}{2} \omega(t)^{2} \vec{r}^{2}$ and interact via time-dependent harmonic interparticle interaction potential of strength $K(t)$ [which depends on $\omega(t)$ and $\delta_{N}$ ]. For the external trapping potential driven by a time-dependent function $f(t)$ :

$$
\omega(t)=\omega_{0}[1+f(t)],
$$

the above imposed requirement $\delta_{N}=\sqrt{\omega_{0}^{2}+2 N K_{0}}=$ const. implies that the interparticle interaction strength has to be driven with the "compensating" time dependency:

$$
K(t)=K_{0}\left[1-\frac{\omega_{0}^{2}}{2 N K_{0}} f(t)\right] .
$$

Since the Hamiltonian (8) or (9) is now time dependent, the total energy is, of course, no longer conserved.

Let us consider a situation where the medium representing $N-1$ relative particles is in the ground state of the harmonic potential with frequency $\delta_{N}=\sqrt{\omega_{0}^{2}+2 N K_{0}}$. Its energy is the time-independent constant $\frac{D}{2}(N-1) \delta_{N}$. The time dependency of the full problem then originates from the driving of the center of mass:

$$
\begin{aligned}
\hat{H}_{\mathrm{c} . \mathrm{m} .} \psi\left(\overrightarrow{q_{N}}, t\right) & =-\frac{1}{2} \partial_{\overrightarrow{q_{N}}}^{2} \psi\left(\overrightarrow{q_{N}}, t\right)+\frac{1}{2} \omega_{0}^{2}[1+f(t)]^{2}{\overrightarrow{q_{N}}}^{2} \psi\left(\overrightarrow{q_{N}}, t\right) \\
& =i \frac{\partial}{\partial t} \psi\left(\overrightarrow{q_{N}}, t\right) .
\end{aligned}
$$

The solution $\psi\left(\overrightarrow{q_{N}}, t\right)$ of this one-particle Schrödinger equation can easily be obtained numerically (see Refs. [33,38]). The final expression for the expectation value of the Hamiltonian Eq. (8) or (9) reads:

$$
\left\langle\Psi(t)\left|\hat{H}_{\text {rel }}+\hat{H}_{\text {c.m. }}(t)\right| \Psi(t)\right\rangle=\frac{D}{2}(N-1) \delta_{N}+\epsilon(t),
$$

where $\epsilon(t)=\left\langle\psi\left(\overrightarrow{q_{N}}, t\right)\left|\hat{H}_{\text {c.m. }}(t)\right| \psi\left(\overrightarrow{q_{N}}, t\right)\right\rangle$. Interestingly, the special kind of time dependency used in Eqs. (10) and (11) also implies that the time-dependent part $\epsilon(t)$ of the expectation value of the total Hamiltonian $\hat{H}(t)$ depends neither on the number of particles $N$ nor on the interaction strength $K_{0}$. Thus, the systems with different particle numbers $N$ and 
different interparticle interaction strengths $K_{0}$ possess the same time-dependent fraction.

It is instructive to state here that the time dependencies, Eqs. (10) and (11), can be more general and it is of course not necessary to choose them such that the relative Hamiltonian $\hat{H}_{\text {rel }}$ remains time independent, i.e., keeping $\delta_{N}=$ const. Yet, there is one important advantage to this particular choice: In the center-of-mass frame, Eq. (8), the relative part is known analytically and to solve the problem completely and exactly one needs to integrate only a single one-particle Schrödinger equation, Eq. (12). In contrast, to find the solution in the laboratory frame one has to solve the time-dependent many-boson Schrödinger equation with a time-dependent trap potential and time-dependent interparticle interactions. While the former task is a manageable standard routine, the latter one comprises a very involved and appealing theoretical and numerical problem. The main goal of the present section is to show that the MCTDHB method is capable of tackling time-dependent scenarios numerically exactly even in the most involved setups: time-dependent one-particle potentials $\hat{V}(\vec{r}, t)$ and time-dependent two-body interactions $\hat{W}\left(\vec{r}, \vec{r}^{\prime}, t\right)$.

In what follows, we investigate the dynamics of the one-dimensional HIM system with time-dependent trapping (10) and interaction (11) potentials driven by two different functions:

$$
\begin{aligned}
& f_{1}(t)=0.2 \sin ^{2}(t), \\
& f_{2}(t)=\sin (t) \cos (2 t) \sin (0.5 t) \sin (0.4 t) .
\end{aligned}
$$

The one-body center-of-mass Schrödinger equation (12) with the respective time-dependent potentials is integrated numerically exactly to obtain the corresponding one-body energies $\epsilon_{1}(t), \epsilon_{2}(t)$.

Let us first study the time-dependent HIM system made of $N=10$ bosons with a relatively simple periodic driving function $f_{1}(t)$ and $K_{0}=0.5$. In the lower part of Fig. 4 we plot the time-dependent part $\epsilon_{1}(t)$ of the respective expectation value of the total Hamiltonian $\hat{H}(t)$ computed by using different levels of the $\operatorname{MCTDHB}(M)$ theory. The numerically exact results for $\epsilon_{1}(t)$ depicted by open circles are obtained by solving directly the one-particle time-dependent Schrödinger equation. It is important to notice that the oscillatory motion of the center of mass results is a relatively small contribution to the total energy: the value of $\epsilon_{1}(t)$ is of the order of a single-particle energy.

A close inspection of Fig. 4 shows that the MCTDHB(3) computation, depicted by a dashed-dotted line, follows the exact curve until $t \approx 5$. To describe the exact dynamics for longer propagation times one needs to use higher levels of the $\operatorname{MCTDHB}(M)$ theory, i.e., more time-adaptive orbitals are needed. The MCTDHB(5) result, plotted by a bold-dashed line, is exact until $t \approx 15$, while the $\operatorname{MCTDHB}(6)$ one depicted by a simple dashed line is exact until $t \approx 30$. The doubledashed line depicting the $\mathrm{MCTDHB}(7)$ result reproduces the exact time dependency of the total energy at all the times considered here.

In the context of ultracold physics the Gross-Pitaevskii mean-field theory is considered as one of the main working tools to describe the dynamics of bosonic systems with timedependent traps and time-dependent interactions. In Fig. 4 we

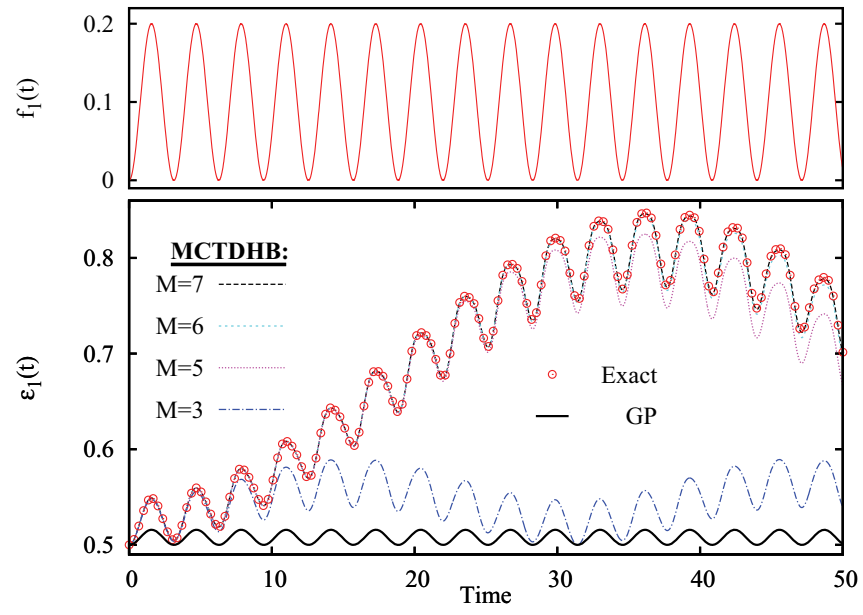

FIG. 4. (Color online) The HIM model with time-dependent trap $V(x)=\omega_{0}[1+f(t)] x^{2}$ and time-dependent interparticle interaction $W\left(x_{i}-x_{j}\right)=K_{0}\left[1-\frac{\omega_{0}^{2}}{2 N K_{0}} f(t)\right]\left(x_{i}-x_{j}\right)^{2}$ permits exact solution. The exact expectation value of the total Hamiltonian of the system, Eq. (13), reads $\langle\Psi(t)|\hat{H}(t)| \Psi(t)\rangle=\epsilon(t)+$ const., with the timeindependent constant equal to $\frac{D}{2}(N-1) \delta_{N}$ and $D=1$. The driving function $f(t)=f_{1}(t)$ and the time-dependent part of the energy $\epsilon(t)=\epsilon_{1}(t)$ for $N=10$ bosons with $K_{0}=0.5$ are plotted. The convergence of $\epsilon_{1}(t)$ when increasing the number of the timeadaptive orbitals $M$ is depicted. The Gross-Pitaevskii results [GP $\equiv$ MCTDHB(1)], plotted by a bold solid line, are inaccurate even for very short time. The MCTDHB(3) provides excellent description up to $t \approx 5$, the $\operatorname{MCTDHB}(5)$ works well till $t \approx 15$, the $\operatorname{MCTDHB}(6)$ till $t \approx 30$, and the $\operatorname{MCTDHB}(7)$ results coincide with the exact solution at all the times depicted. See text for discussion. All quantities shown are dimensionless.

also plot the results obtained by solving the GP equation with the harmonic interaction, which is identical to the lowest level MCTDHB(1) theory, by a bold solid line. The GP theory is incapable of describing the time-dependent energy correctly even for short times. Note that $N=10$ only.

Now we study the HIM systems made of $N=10$ and $N=50$ bosons with $K_{0}=0.5$ driven by quite a complicated time-dependent function $f_{2}(t)$, depicted in the upper part of Fig. 5. The exact $\epsilon_{2}(t)$, obtained by solving the corresponding one-particle Schrödinger equation, is plotted by open red circles. As was discussed above, this time-dependent fraction of the expectation value of the Hamiltonian $\hat{H}(t)$ is the same for both systems. We use different levels of the $\operatorname{MCTDHB}(M)$ theory to compute the time-dependent contribution $\epsilon_{2}(t)$ to the energy of the studied systems. The dashed-dotted and dashed-double-dotted lines are used, respectively, to depict the $\operatorname{MCTDHB}(M=6)$ and $\operatorname{MCTDHB}(M=7)$ results for the system with $N=10$ bosons. The $\operatorname{MCTDHB}(M=5)$ and $\operatorname{MCTDHB}(M=6)$ results for the system made of $N=$ 50 bosons are depicted by the dashed and bold-dashed lines, correspondingly. All the presented numerical MCTDHB results with $M>5$ follow the exact lines till $t \approx 25$, indicating that numerical convergence is reached. In Fig. 5 we also depict the corresponding Gross-Pitaevskii results. The GP theory, usually considered to be applicable for systems made of a larger number of particles, provides a semiqualitative 

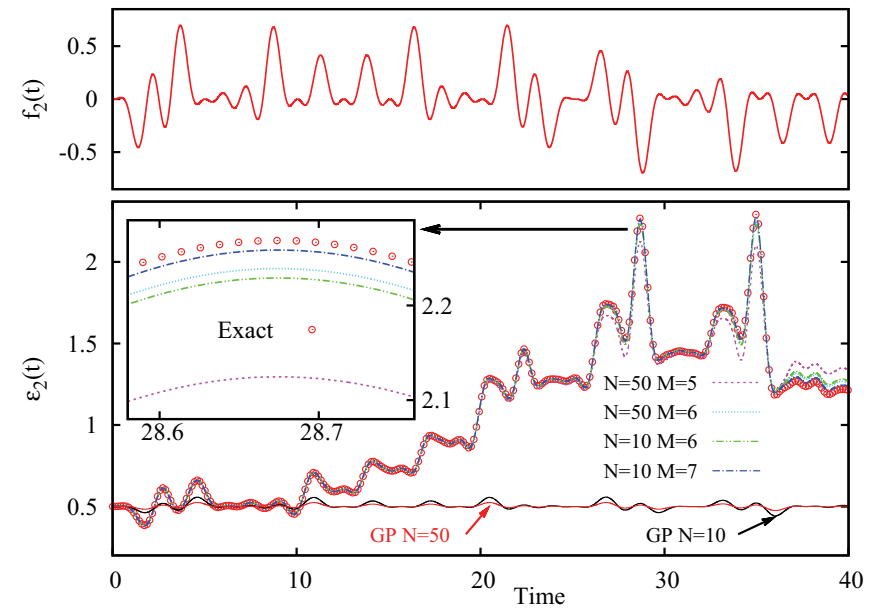

FIG. 5. (Color online) The modified HIM model with timedependent trap and interparticle interaction driven by a complicated function $f_{2}(t)$ [Eq. (14)]. The function is depicted in the upper panel. The time-dependent contribution $\epsilon_{2}(t)$ to the total energies is computed at several different levels of the $\operatorname{MCTDHB}(M)$ theory for $N=10[M=6,7]$ and $N=50[M=5,6]$ bosons. The strength of the interparticle interaction is $K_{0}=0.5$. The considered time dependency of the one- and two-body interaction potentials guarantees that the exact $\epsilon_{2}(t)$, plotted by open red circles, is the same for both systems. The MCTDHB(5) for $N=50$ and $\operatorname{MCTDHB}(6)$ for $N=10$ provide a converged description of the dynamics till $t \approx 25$; for longer times more orbitals are needed for numerical exactness. The corresponding Gross-Pitaevskii results, marked by arrows, are semiqualitative for very short initial times only, till $t \approx 1$. See text for discussion. All quantities shown are dimensionless.

description of the very short initial dynamics $(t \approx 1)$; afterwards the quality of its predictions sharply deteriorates.

Summarizing, the $\operatorname{MCTDHB}(M)$ computations with a given number of time-adaptive orbitals start to deviate from the exact result with time (see Fig. 5 and its inset). The time-dependent variational principle used in the MCTDHB method implies that the MCTDHB computations done with a larger number of the time-adaptive orbitals remain "on top" of the exact curve for longer propagation times. Even when the numerical many-body results slightly deviate from the exact at longer propagation times they are quantitative and quite accurate-all the spectral features of the exact behavior are reproduced (see Fig. 5). In conclusion, the MCTDHB method is capable of providing numerically exact results for timedependent Hamiltonians with very general driving scenarios, where both the external trap and interparticle interactions are driven in quite a complicated way.

\section{DISCUSSION AND OUTLOOK}

In the present work we have compared the quantum many-boson physics of the HIM system described in the laboratory and in the center-of-mass frames. In contrast to the center-of-mass frame where the HIM problem is exactly solvable, in the laboratory frame one has to invest numerical efforts to reproduce the exact results, even in the two-particle case. The relevance of self-consistency and time adaptivity is demonstrated. To solve time-independent problems, the standard many-body full configuration interaction (exact diagonalization) method requires one to use a large number of fixed-shape (nonoptimal) one-particle basis functions, thereby restricting its applicability to few-particle systems. The usage of the MCTDHB method utilizing variational self-consistent basis sets allows one to attack systems with larger particle numbers. To verify how good the obtained static solution is one can use the straightforward methodology: by comparing the eigenstates obtained by imaginary time propagation of the MCTDHB equations with $M$ and $M+1$ orbitals one can conclude that numerical exactness is achieved. See Table I for reference.

To check the relevance of the time adaptivity we have first studied the time-dependent HIM problem where the dynamics are initiated by a sudden quench of the interparticle interaction strength from zero to some finite value. It has been shown that the many-body method (MCTDHB) utilizing variationally optimal time-adaptive orbitals allows one to obtain the numerically exact solutions for much longer propagation times in comparison to the fixed-orbital FCI (ED) method spanning a Fock space of the same size. The methodology in determining the accuracy of the time-dependent solution obtained is to compare the properties of the MCTDHB solutions computed by using $M$ and $M+1$ time-adaptive orbitals at different propagation times. If more time-adaptive orbitals are used than needed, the Dirac-Frenkel variational principle keeps the superfluous orbitals unoccupied, i.e., they do not contribute to the now converged and exact many-boson wave function. Generally, we have found that one needs less time-adaptive orbitals to converge the results for bosonic systems with increasing number $N$ of particles when the interaction strength $\Lambda=K_{0}(N-1)$ is kept fixed.

In the broader context, several other methods of the family of the multiconfigurational methods have been benchmarked in the field. The first of these, the multiconfigurational timedependent Hartree (MCTDH, MCTDHB's mother method) [39-41], was benchmarked with standard wave-packet propagation [39-41] as well as with experimental spectra (see, e.g., Refs. [42,43]). The MCTDH for fermions (MCTDHF, a sister method of MCTDHB) [44-47] was benchmarked with direct numerical solutions of the Schrödinger equation (see, e.g., Refs. $[48,49]$ ). Similarly, the aim of the present study was to benchmark and assess the properties of the convergence of MCTDHB with respect to the number of variational parameters used. Throughout this work, the MCTDHB method has been benchmarked with the standard HIM. The convergence of the ground state and nonequilibrium dynamics has been demonstrated. We prove, thereby, that the MCTDHB can be used to obtain numerically exact solutions of the many-boson TDSE.

We have also shown that the exactly solvable many-body HIM problem can be extended to a driven time-dependent Hamiltonian. Namely, if the time-dependent modulation of the harmonic trap is accompanied by the modulation of the interparticle interaction with the same driving function (with a different amplitude) all the internal excitations in such a system can be compensated and the many-body system behaves as a driven single-particle system. For systems with large particle numbers this driving contribution is of the order of a singleparticle energy. Physically, it means that the modulation of the 
harmonic trap can be almost completely compensated by the corresponding modulation of the interparticle interaction.

The driving scenario proposed for the HIM is based on the separability of the relative and center-of-mass coordinates and, therefore, it can also be adapted to other many-body systems with such a separability. In particular, it can work in many-body systems trapped in harmonic potentials and interacting via other two-body potentials which depend on the interparticle separation. However, it should be noted that, whereas the "compensating" relation between the trap and interparticle modulations are of simple form for harmonic interactions [see Eqs. (10) and (11)], in the case of ultracold gases (with contact interactions) this relation is expected to be much more involved. Summarizing, in trapped manyparticle systems where the center of mass is separable, a novel phenomenon of "dynamical compensation" can take place - all the excitations originating from a driven trapping potential can be almost completely dynamically compensated by the respective driving of the interparticle interaction potential.

The driven many-body system can, in principle, be realized in the context of ultracold physics. It would correspond to an experimental setup where the trap potential (magneto-optical trap) and external magnetic field, used for the Feshbach resonance technique, are driven such that the relative phase and amplitude of the time-dependent modulations can be tuned. By measuring, e.g., the density response as a function of the amplitude of the modulation applied, one can scan for and verify the predicted effect. If the dynamical compensation does not take place, more and more excited states would contribute to the dynamics, and in time the density will oscillate with larger and larger amplitude. On the contrary, when the compensation is achieved the density response to the applied modulations remains very weak even for long exposition times. It is important to note that this prediction is valid for many-boson systems where the relative motion is not only in the ground but also in excited states. In particular, it means that the dynamical compensation could work at nonzero temperatures as well.

\section{ACKNOWLEDGMENTS}

Computation time on the bwGRiD and the Cray XE6 cluster "Hermit" at the HLRS in Stuttgart, and financial support by the HGS MathComp, Minerva Short Term research grant, and the DFG also within the framework of the "Enable fund" of the excellence initiative at Heidelberg University are greatly acknowledged.
[1] W. Ketterle, Rev. Mod. Phys. 74, 1131 (2002).

[2] E. Cornell and C. Wieman, Rev. Mod. Phys. 74, 875 (2002).

[3] C. C. Bradley, C. A. Sackett, J. J. Tollett, and R. G. Hulet, Phys. Rev. Lett. 75, 1687 (1995).

[4] A. Görlitz, J. M. Vogels, A. E. Leanhardt, C. Raman, T. L. Gustavson, J. R. Abo-Shaeer, A. P. Chikkatur, S. Gupta, S. Inouye, T. Rosenband, and W. Ketterle, Phys. Rev. Lett. 87, 130402 (2001).

[5] F. Schreck, L. Khaykovich, K. L. Corwin, G. Ferrari, T. Bourdel, J. Cubizolles, and C. Salomon, Phys. Rev. Lett. 87, 080403 (2001).

[6] M. Greiner, I. Bloch, O. Mandel, T. W. Hänsch, and T. Esslinger, Phys. Rev. Lett. 87, 160405 (2001).

[7] Ch. Chin, R. Grimm, P. Julienne, and E. Tiesinga, Rev. Mod. Phys. 82, 1225 (2010).

[8] K. Henderson, C. Ryu, C. MacCormick, and M. G. Boshier, New J. Phys. 11, 043030 (2009).

[9] A. Griesmaier, J. Werner, S. Hensler, J. Stuhler, and T. Pfau, Phys. Rev. Lett. 94, 160401 (2005).

[10] J. Stuhler, A. Griesmaier, T. Koch, M. Fattori, T. Pfau, S. Giovanazzi, P. Pedri, and L. Santos, Phys. Rev. Lett. 95, 150406 (2005).

[11] M. Lu, N. Q. Burdick, S. H. Youn, and B. L. Lev, Phys. Rev. Lett. 107, 190401 (2011).

[12] L. Cohen and C. Lee, J. Math. Phys. 26, 3105 (1985).

[13] J. Yan, J. Stat. Phys. 113, 623 (2003).

[14] A. I. Streltsov, O. E. Alon, and L. S. Cederbaum, Phys. Rev. Lett. 99, 030402 (2007).

[15] O. E. Alon, A. I. Streltsov, and L. S. Cederbaum, Phys. Rev. A 77, 033613 (2008).
[16] N. K. Wilkin, J. M. F. Gunn, and R. A. Smith, Phys. Rev. Lett. 80, 2265 (1998).

[17] M. A. Załuska-Kotur, M. Gajda, A. Orłowski, and J. Mostowski, Phys. Rev. A 61, 033613 (2000).

[18] M. Gajda, Phys. Rev. A 73, 023603 (2006).

[19] A. I. Streltsov, K. Sakmann, A. U. J. Lode, O. E. Alon, and L. S. Cederbaum, The Multiconfigurational Time-Dependent Hartree for Bosons Package, version 2.1, Heidelberg, http://mctdhb.org.

[20] A. I. Streltsov, O. E. Alon, and L. S. Cederbaum, Phys. Rev. Lett. 100, 130401 (2008).

[21] K. Sakmann, A. I. Streltsov, O. E. Alon, and L. S. Cederbaum, Phys. Rev. Lett. 103, 220601 (2009).

[22] K. Sakmann, A. I. Streltsov, O. E. Alon, and L. S. Cederbaum, Phys. Rev. A 82, 013620 (2010).

[23] A. I. Streltsov, O. E. Alon, and L. S. Cederbaum, Phys. Rev. Lett. 106, 240401 (2011).

[24] A. I. Streltsov, K. Sakmann, O. E. Alon, and L. S. Cederbaum, Phys. Rev. A 83, 043604 (2011).

[25] J. Grond, J. Schmiedmayer, and U. Hohenester, Phys. Rev. A 79, 021603(R) (2009).

[26] J. Grond, T. Betz, U. Hohenester, N. J. Mauser, J. Schmiedmayer, and T. Schumm, New J. Phys. 13, 065026 (2011).

[27] J. Grond, U. Hohenester, J. Schmiedmayer, and A. Smerzi, Phys. Rev. A 84, 023619 (2011).

[28] I. Březinová, A. U. J. Lode, A. I. Streltsov, O. E. Alon, L. S. Cederbaum, and J. Burgdörfer, Phys. Rev. A 86, 013630 (2012).

[29] A. I. Streltsov, O. E. Alon, and L. S. Cederbaum, Phys. Rev. A 73, 063626 (2006).

[30] F. Dalfovo, S. Giorgini, L. P. Pitaevskii, and S. Stringari, Rev. Mod. Phys. 71, 463 (1999).

[31] A. J. Leggett, Rev. Mod. Phys. 73, 307 (2001). 
[32] C. J. Pethick and H. Smith, Bose-Einstein Condensation in Dilute Gases, 2nd ed. (Cambridge University Press, Cambridge, England, 2008).

[33] W. H. Press, S. A. Teukolsky, W. T. Vetterling, and B. P. Flannery, Numerical Recipes in Fortran (Cambridge University Press, Cambridge, England, 1992).

[34] T. J. Park and J. C. Light, J. Chem. Phys 85, 5870 (1986).

[35] Z. Bačić and J. C. Light, J. Chem. Phys 85, 4594 (1986).

[36] K. Sakmann, A. I. Streltsov, O. E. Alon, and L. S. Cederbaum, New J. Phys. 13, 043003 (2011).

[37] Wolfram Research, Inc., MATHEMATICA, Version 8.0, Champaign, IL (2010).

[38] R. Kosloff, J. Phys. Chem. 92, 2087 (1988).

[39] H.-D. Meyer, U. Manthe, and L. S. Cederbaum, Chem. Phys. Lett. 165, 73 (1990).

[40] U. Manthe, H.-D. Meyer, and L. S. Cederbaum, J. Chem. Phys. 97, 3199 (1992).
[41] Multidimensional Quantum Dynamics: MCTDH Theory and Applications, edited by H.-D. Meyer, F. Gatti, and G. A. Worth (Wiley-VCH, Weinheim, 2009).

[42] A. Raab, G. A. Worth, H.-D. Meyer, and L. S. Cederbaum, J. Chem. Phys 110, 936 (1999).

[43] O. Vendrell, M. Brill, F. Gatti, D. Lauvergnat, and H.-D. Meyer, J. Chem. Phys. 130, 234305 (2009).

[44] J. Zanghellini, M. Kitzler, C. Fabian, T. Brabec, and A. Scrinzi, Laser Phys. 13, 1064 (2003).

[45] J. Caillat, J. Zanghellini, M. Kitzler, O. Koch, W. Kreuzer, and A. Scrinzi, Phys. Rev. A 71, 012712 (2005).

[46] T. Kato and H. Kono, Chem. Phys. Lett. 392, 533 (2004).

[47] M. Nest, T. Klamroth, and P. Saalfrank, J. Chem. Phys. 122, 124102 (2005).

[48] D. Hochstuhl, S. Bauch, and M. Bonitz, J. Phys.: Conf. Ser. 220, 012019 (2010).

[49] D. Hochstuhl and M. Bonitz, J. Chem. Phys. 134, 084106 (2011). 